13

\title{
Нелинейное подавление сигналов в лампе бегущей волны
}

\author{
(ㄷ С.В. Гришин, Б.С. Дмитриев, Ф.П. Разуваев, В.Н. Скороходов, В.Н. Титов, Д.И. Трубецков
}

Саратовский национальный исследовательский государственный университет им. Н.Г. Чернышевского, 410012 Саратов, Россия

e-mail: sergrsh@yandex.ru

Поступило в Редакцию 28 апреля 2021 г.

В окончательной редакции 18 июня 2021 r.

Принято к публикации 20 июня 2021 г.

\begin{abstract}
Представлены результаты экспериментального и теоретического исследований прохождения монохроматического и импульсно-модулированного сверхвысокочастотных сигналов через лампу бегущей волны, работающую в режиме нелинейного подавления. Установлено, что в зависимости от величины ускоряющего напряжения и тока пучка лампа может работать либо как насыщающийся поглотитель, либо как ограничитель мощности. В первом случае максимальный уровень подавления сигнала, соответствующий срыву Компфнера, реализуется в линейном режиме, а во втором случае - в нелинейном режиме, при определенной мощности входного сигнала. Показано, что в случае максимального подавления на фронте радиоимпульсов формируется короткий импульс. В режиме нелинейного срыва Компфнера длительность такого импульса уменьшается с увеличением мощности входного радиоимпульса. Результаты расчета, полученные по нелинейной нестационарной теории лампы бегущей волны, находятся в хорошем качественном соответствии с экспериментальными данными.
\end{abstract}

Ключевые слова: лампа бегущей волны, срыв Комфнера, насыщающийся поглотитель, ограничитель мощности.

DOI: 10.21883/JTF.2021.11.51543.129-21

\section{Введение}

Лампа бегущей волны (ЛБВ) - является первым широкополосным вакуумным усилителем, работающим в микроволновом диапазоне, который был разработан А. Гаевым и Р. Комфнером независимо друг от друга [1]. Работа ЛБВ как усилителя сверхвысокочастотного (СВЧ) сигнала реализуется, как правило, при положительных значениях параметра несинхронности, когда скорость электронов немного превосходит фазовую скорость электромагнитной волны, распространяющейся вдоль замедляющей системы (3С). В этом случае электронные сгустки формируются в области тормозящих фаз поля, что обеспечивает передачу энергии от потока электронов бегущей электромагнитной волне и ее усиление. Однако в ЛБВ возможно реализовать и другую ситуацию, когда при определенных значениях тока пучка и ускоряющего напряжения скорость электронов становится немного меньше фазовой скорости электромагнитной волны. Это приводит к тому, что при отрицательных значениях параметра несинхронности электроны оказываются в области ускоряющих фаз поля, и энергия электромагнитной волны тратится на их ускорение. В этом случае ЛБВ работает уже не как усилитель, а как подавитель СВЧ сигнала.

Режим бесконечного затухания или полного подавления СВЧ сигнала реализуется при некоторых строго определенных значениях потенциала и тока пучка ЛБВ, которые получили в литературе название условий срыва Компфнера (Kompfner dip conditions) [2-4]. В отсут- ствие затухания и в пренебрежении пространственным зарядом упомянутые выше условия имеют следующий вид: $C N=0.315, \Phi_{0}=\pi \quad\left(\right.$ где $C=\left[I_{d} R_{c} /\left(4 U_{d}\right)\right]^{1 / 3}-$ параметр усиления Пирса, $I_{d}$ и $U_{d}$ - ток и напряжение пучка, соответствующие бесконечному затуханию волны, $R_{c}$ - сопротивление связи пучка и волны, $N$ - электрическая длина пространства взаимодействия и $\Phi_{0}$ невозмущенный относительный угол пролета электронов) [4]. ЛБВ подавитель, работающий в режиме срыва Компфнера, использовался в основном для определения сопротивления связи и дисперсии ЗС [5]. Преимущества использования срыва Комфнера для определения электродинамических характеристик ЗС связаны прежде всего с тем, что условия бесконечного затухания СВЧ сигнала очень чувствительны к величине потенциала и тока пучка. Малейшее отклонение от их критических значений $\left(I_{d}\right.$ и $\left.U_{d}\right)$ приводит к появлению СВЧ сигнала на выходе ЛБВ. Кроме того, для определения указанных выше электродинамических характеристик 3С можно не учитывать согласование 3С с подводящим трактом, так как явление срыва Компфнера объясняется интерференцией электромагнитной волны, подаваемой на вход ЛБВ, и волны, наводимой в системе электронным потоком. Явление бесконечного затухания волны использовалось также в параметрических приборах для перекачки энергии от структуры к пучку. Устройство со спиральной замедляющей системой, применяемое для этих целей, получило название преобразователя Компфнера [6,7]. Однако во всех перечисленных выше работах исследования проводились в линейном режиме ЛБВ. 
Первые теоретические и экспериментальные исследования условий бесконечного затухания СВЧ сигнала в нелинейном режиме работы ЛБВ подавителя были проведены в Саратовском государственном университете в середине 60-х годов прошлого столетия $[8,9]$. На основе аппроксимации уравнений линейной теории, полученных методом последовательных приближений, в нелинейную область были получены приближенные условия полного подавления сигнала в нелинейном режиме работы ЛБВ. Экспериментальные исследования влияния уровня входной мощности $\left(P_{i n}\right)$ на условия бесконечного затухания сигнала были проведены на ЛБВ дециметрового диапазона со спиральной ЗС и заключались в изучении зависимостей $I_{d}, U_{d}$ от $P_{\text {in }}$ и сопоставления этих зависимостей с рассчитанными по нелинейной одномерной модели в отсутствие затухания и в пренебрежении пространственным зарядом. В [10] сообщалось об использовании режима полного подавления СВЧ сигнала для создания разнообразных конструкций широкополосных ЛБВ-вентилей, которые использовались в усилительных цепочках на входе мощных прозрачных ЛБВ усилителей для подавления отраженного от их входа СВЧ сигнала.

Интерес к нелинейному режиму работы ЛБВ, работающей в условиях срыва Компфнера, возобновился в последние годы, когда группой российских ученых было предложено использовать ЛБВ подавитель в качестве насыщающегося поглотителя, у которого, по аналогии с оптическими системами, величина подавления сигнала зависит от уровня его мощности (интенсивности) [11-14]. Было установлено, что для монохроматического СВЧ сигнала большого уровня мощности подавление является минимальным. Оно возрастает с уменьшением уровня $P_{\text {in }}$ и насыщается, достигая максимального значения для СВЧ сигнала малого уровня мощности. В теоретических работах [11-13] на основе разработанных численных нелинейных моделей была продемонстрирована возможность использования компфнеровских поглотителей в цепи обратной связи кольцевого автогенератора для формирования мощных ультракоротких импульсов. Экспериментальная реализация пассивной синхронизации мод с помощью ЛБВподавителя, работающего в режиме срыве Компфнера, была осуществлена в работе [14].

Описанные выше импульсные режимы были получены, когда максимальный уровень подавления монохроматического СВЧ сигнала достигался в линейном режиме работы ЛБВ-подавителя. В настоящей работе демонстрируется возможность формирования коротких импульсов с длительностями порядка нескольких наносекунд вне кольцевой генераторной схемы, когда максимальный уровень подавления сигнала реализуется либо в линейном, либо в нелинейном режиме работы ЛБВ подавителя.

\section{1. Экспериментальные результаты}

\section{1. Нелинейное подавление монохроматического СВЧ сигнала}

Эксперименты проводились с прозрачной ЛБВ, выполненной на основе регулярной спиральной $3 \mathrm{C}$ без поглотителя. Лампа работала в дециметровом диапазоне на частотах от 600 до $1130 \mathrm{MHz}$. Коэффициент усиления лампы достигал значения $\sim 20 \mathrm{~dB}$ на частоте $800 \mathrm{MHz}$ при токе пучка $I_{0}=60 \mu \mathrm{A}$ и ускоряющем напряжении $U_{0}=117 \mathrm{~V}$. Длина однородного участка спирали 3С $186 \mathrm{~mm}$, шаг спирали - $0.27 \mathrm{~mm}$, диаметр витка спирали $-3.6 \mathrm{~mm}$, диаметр провода спирали $-0.2 \mathrm{~mm}$, материал спирали - молибден. Для измерения S-параметров ЛБВ использовался анализатор цепей, обеспечивающий изменение уровня мощности монохроматического СВЧ сигнала на входе ЛБВ в диапазоне от -30 до $0 \mathrm{dBm}$. Измерения модуля коэффициента передачи $K$ проводились как в режиме свипирования частоты $f$ при фиксированном значении входной мощности $P_{i n}$, так и в режиме свипирования входной мощности на фиксированной частоте.

На рис. 1 приведены АЧХ ЛБВ-подавителя, полученные при условии, что центральная частота полосы подавления $f_{0}$ находится в центральной части рабочей полосы частот лампы. Измерения проводились для разных значений тока пучка, ускоряющего напряжения и входной мощности. Из представленных на рис. 1, $a$ результатов следует, что при малых уровнях $P_{\text {in }}$ на АЧХ лампы присутствует относительно узкий провал, на центральной частоте которого $f_{0} \cong 800 \mathrm{MHz}$ наблюдается практически полное подавление монохроматического СВЧ сигнала. При выбранных значениях тока пучка и ускоряющего напряжения электронный пучок попадает в область максимального ускоряющего поля, забирая практически всю энергию от электромагнитной волны. В этом случае реализуется режим срыва Компфнера [2-4]. Увеличение уровня $P_{\text {in }}$ приводит к уменьшению уровня подавления сигнала на частоте $f_{0}$, что обусловлено уменьшением скорости электронов из-за их торможения бегущей волной. В этом случае электроны начинают уходить из области максимального ускоряющего поля, не отбирая всю энергию от электромагнитной волны. Как следует из результатов, представленных на рис. 1, $c$ (кривая 5), на зависимости модуля коэффициента передачи от входной мощности $K\left(P_{i n}\right)$ наблюдаются два участка, один из которых соответствует линейному режиму работы ЛБВ, а другой - ее нелинейному режиму работы. Нелинейный участок начинается при $P_{t h 0}=-26.3 \mathrm{dBm}$, когда зависимость $K\left(P_{i n}\right)$ отклоняется от линейной на $1 \mathrm{~dB}$. Возникновение нелинейного участка обусловлено уменьшением скорости электронов с ростом амплитуды электромагнитной волны. При $P_{i n}>P_{t h 0}$ коэффициент передачи возрастает, a при достаточно больших уровнях входной мощно- 

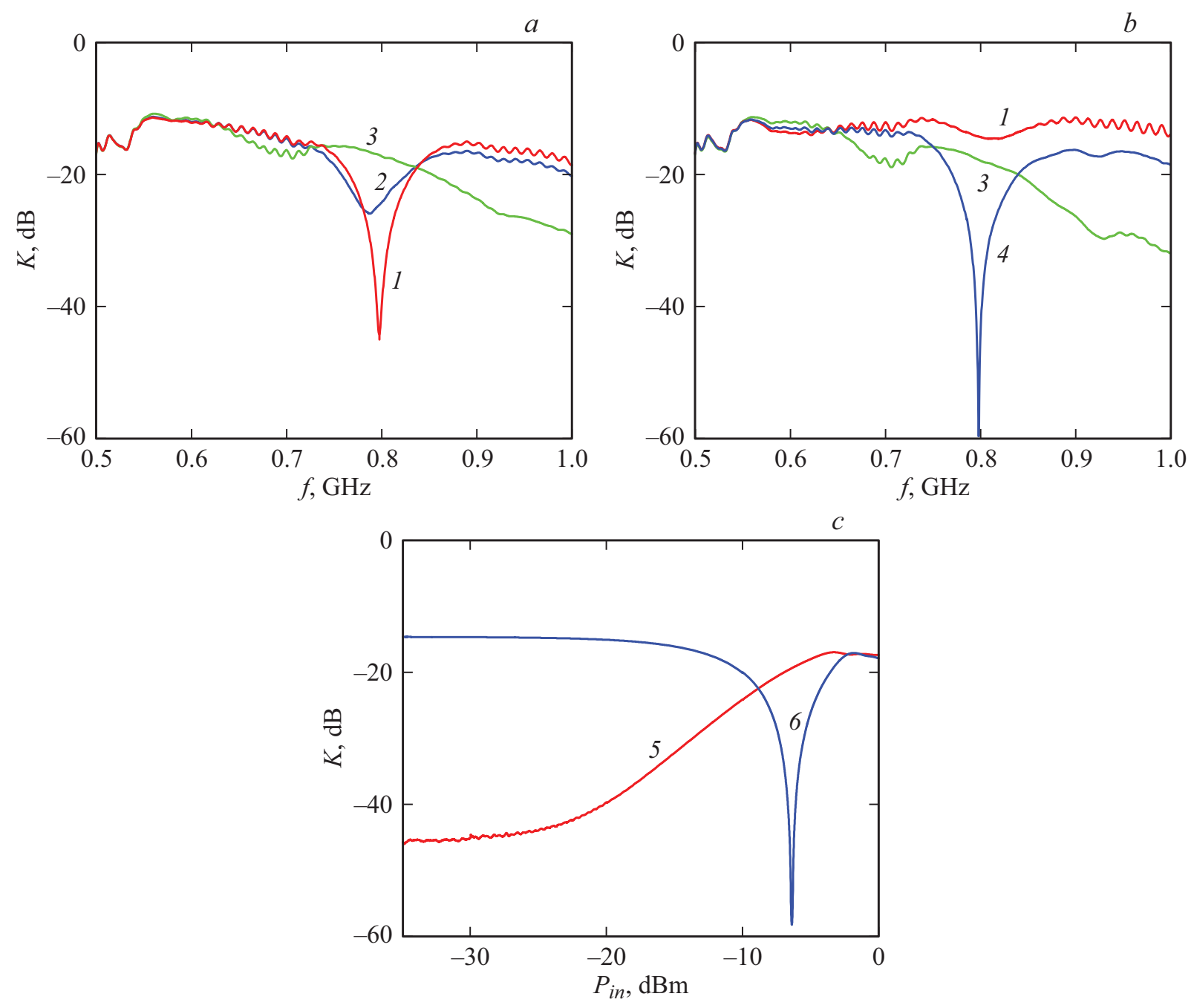

Рис. 1. $a, b-\mathrm{AЧХ} \mathrm{ЛБВ} \mathrm{подавителя,} \mathrm{измеренные} \mathrm{в} \mathrm{режиме} \mathrm{срыва} \mathrm{Компфнера}\left(I_{0}=3 \mu \mathrm{A}, U_{0}=108 \mathrm{~V}\right)(a)$ и в режиме нелинейного срыва Компфнера $\left(I_{0}=10 \mu \mathrm{A}, U_{0}=107 \mathrm{~V}\right)(b)$, при разных уровнях входной мощности $P_{i n}:-30 \mathrm{dBm}($ кривая 1$),-10 \mathrm{dBm}$ (кривая 2), $0 \mathrm{dBm}$ (кривая 3) $-6.4 \mathrm{dBm}$ (кривая 4); зависимости модуля коэффициента передачи $K$ от мощности сигнала на выходе ЛБВ-подавителя $P_{i n}$, измеренные на частоте $f_{0}$ в режиме срыва Компфнера (кривая 5 ), и в режиме нелинейного срыва Компфнера (кривая 6) (c).

сти достигает максимального значения. В этом случае ЛБВ-подавитель работает как насыщающийся поглотитель $[11,14]$.

При других значениях тока пучка и ускоряющего напряжения (рис. $1, b$ ) ситуация меняется кардинальным образом. Здесь при малых уровнях $P_{\text {in }}$ на АЧХ лампы не наблюдается полного подавления монохроматического СВЧ сигнала. Практически полное подавление сигнала достигается на частоте $f_{0}$ только в нелинейном режиме при значении входной мощности $P_{i n}=-6.4 \mathrm{dBm}$. Однако при уровнях мощности $P_{i n}>-6.4 \mathrm{dBm}$ оно опять уменьшается. Таким образом, при $P_{\text {in }}=-6.4 \mathrm{dBm}$ наблюдается режим практически полного подавления монохроматического сигнала, аналогичный классическому срыву Компфнера, с той лишь разницей, что наибольшее подавление наблюдается на частоте $f_{0}$ не только за счет выбора соответствующих значений тока пучка и ускоряющего напряжения, но и за счет выбора амплитуды бегущей электромагнитной волны. Как следует из результатов, представленных на рис. 1, с (кривая 6), на зависимости $K\left(P_{\text {in }}\right)$ также присутствуют два участка: линейный и нелинейный. Однако, в отличие от результатов, представленных на рис. 1,c (кривая 5), здесь имеются два характерных уровня мощности, один из которых $P_{t h 1}=-16.7 \mathrm{dBm}$ соответствует началу нелинейного режима работы лампы, а другой $P_{t h 2}=-6.4 \mathrm{dBm}$ соответствует максимальному уровню подавления сигнала в нелинейном режиме. Если предположить, что, как и в предыдущем случае, основное влияние на скорость движения электронов в нелинейном режиме оказывает амплитуда бегущей волны, то на качественном уровне получение максимального уровня подавления сигнала при определенном уровне его входной мощности можно трактовать следующим образом. В линейном режиме, 
когда $P_{i n}<P_{t h 1}$, электронный пучок при выбранных значениях тока и напряжения не находится долгое время в области максимального ускоряющего поля. Увеличение мощности входного сигнала приводит к формированию электронного сгустка, который находится в ускоряющем поле существенную часть времени движения вдоль ЗС. Таким образом, в интервале значений входной мощности $P_{t h 1}<P_{i n}<P_{t h 2}$ нарастание амплитуды бегущей волны приводит к ускорению электронов, которые при взаимодействии с бегущей волной приобретают большую энергию. Это обусловливает ограничение мощности СВЧ сигнала на выходе ЛБВ подавителя. При $P_{i n}=P_{t h 2}$ сформировавшийся электронный сгусток находится долгое время в области максимального ускоряющего поля, забирая практически всю энергию от электромагнитной волны. При $P_{t h}>P_{t h 2}$ мощность СВЧ сигнала на выходе ЛБВ подавителя начинает возрастать из-за того, что электронный сгусток находится теперь долгое время в области тормозящего поля, отдавая свою энергию электромагнитной волне. Однако на обоих нелинейных участках зависимости $K\left(P_{i n}\right)$ значения модуля коэффициента передачи не превышают значений, полученных в линейном режиме. Таким образом, ЛБВ подавитель при выбранных значениях тока пучка, ускоряющего напряжения и мощности входного СВЧ сигнала работает как ограничитель мощности.

Необходимо отметить, что в эксперименте для выбранных значений тока и напряжения пучка пороговые уровни мощности $P_{t h 0}$ и $P_{t h 1}$, соответствующие началу нелинейного режима работы лампы в двух разных режимах подавления, отличаются друг от друга на величину $\sim 10 \mathrm{~dB}$. Кроме того, как следует из результатов, представленных на рис. $1, a, b$, наибольшие уровни подавления СВЧ сигнала удается получить в режиме нелинейного срыва Компфнера, хотя в теории бесконечное затухание СВЧ сигнала должно наблюдаться и в линейном режиме. Данный факт обусловлен тем, что при проведении экспериментальных исследований более высокие уровни подавления сигнала, наблюдаемые в режиме нелинейного срыва Компфнера, обеспечиваются за счет более плавной перестройки входной мощности сигнала, которая изменялась с шагом $0.1 \mathrm{~dB}$. В режиме срыва Компфнера изменение тока пучка осуществлялось с шагом $1 \mu \mathrm{A}$, что было недостаточно для получения большего уровня подавления сигнала.

\section{2. Нелинейное подавление импульсно-модулированного СВЧ сигнала}

Были проведены экспериментальные исследования прохождения импульсно-модулированного (ИМ) СВЧ сигнала через ЛБВ подавитель. Частота несущей ИМ СВЧ сигнала соответствовала частоте $f_{0}$, на которой наблюдалось практически полное подавление монохроматического СВЧ сигнала либо в линейном режиме (срыв Компфнера), либо в нелинейном режиме (нелинейный срыв Компфнера). ИМ СВЧ сигнал, посту- павший на вход ЛБВ-подавителя, представлял собой периодическую последовательность радиоимпульсов с несущей частотой $f_{0}$, длительностью $3 \mu \mathrm{s}$ и скважностью $q=2$. Длительность радиоимпульсов выбиралась, исходя из того, чтобы спектр ИМ СВЧ сигнала был относительно узкополосным и находился в полосе частот срыва Компфнера. Для измерения временных рядов использовался осциллограф реального времени с полосой пропускания $10 \mathrm{GHz}$ и частотой дискретизации $20 \mathrm{Gs} / \mathrm{s}$.

На рис. 2 и 3 приведены огибающие радиоимпульсов, измеренные как на входе, так и на выходе ЛБВ, работающей в одном из двух режимов подавления. Из представленных результатов следует, что в обоих режимах, когда подавление монохроматического СВЧ сигнала является максимальным, основная часть радиоимпульса практически полностью подавляется (рис. 2, $a$ и 3,b). ${ }^{1}$ Однако на фронте и срезе огибающей радиоимпульса остаются „выплески“ в виде относительно коротких импульсов огибающей, длительность которых является намного меньше длительности исходного радиоимпульса. Необходимо отметить, что аналогичное поведение огибающей радиоимпульса наблюдается при прохождении последнего через режекторный фильтр, свойствами которого в данном случае обладает ЛБВ-подавитель, работающий либо в режиме срыва Компфнера (рис. 2), либо в режиме нелинейного срыва Компфнера (рис. 3). В первом случае, при $P_{p} \sim P_{t h 0}$ (рис. $\left.2, a\right)$, длительность короткого импульса огибающей, находящегося на фронте исходного радиоимпульса, имеет величину $\sim 6 \mathrm{~ns}$, которая измерена по половинному уровню от максимального значения амплитуды. Во втором случае, при $P_{p}=P_{t h 2}($ рис. $3, b)$, длительность короткого импульса огибающей имеет величину $\sim 11 \mathrm{~ns}$, которая практически в два раза больше той, которая была получена в условиях срыва Компфнера.

Дальнейшее увеличение пиковой мощности входных радиоимпульсов приводит к отличному поведению коротких импульсов огибающей для двух рассматриваемых режимов нелинейного подавления. Как следует из результатов, представленных на рис. $2, b-d$, в режиме срыва Компфнера увеличение пиковой мощности входных радиоимпульсов приводит к уменьшению уровня подавления основной части выходного радиоимпульса, амплитуда которой растет быстрее пиковых амплитуд коротких импульсов огибающей. Увеличение пиковой мощности входного радиоимпульса в три раза (рис. $2, b$ ) приводит к небольшому увеличению длительности короткого импульса огибающей на фронте выходного радиоимпульса до величины $\sim 7 \mathrm{~ns}$. Увеличение пиковой мощности входного радиоимпульса практически

\footnotetext{
${ }^{1}$ На рис. 2, a результаты получены при $P_{p}>P_{t h 0}$, так как при $P_{p} \leq P_{t h 0}$ радиоимпульсы, проходя через ЛБВ подавитель и испытывая сильное ослабление, не регистрируются на экране осциллографа. Однако, как следует из результатов, представленных на рис. $1, c$, при $P_{p}=-20 \mathrm{dBm}$ уровень подавления сигнала все еще остается близким к уровню максимального компфнеровского подавления.
} 
$a$
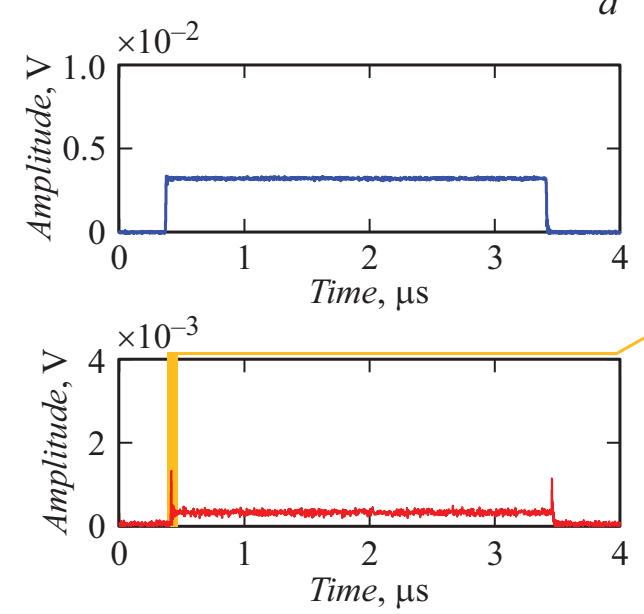

$c$
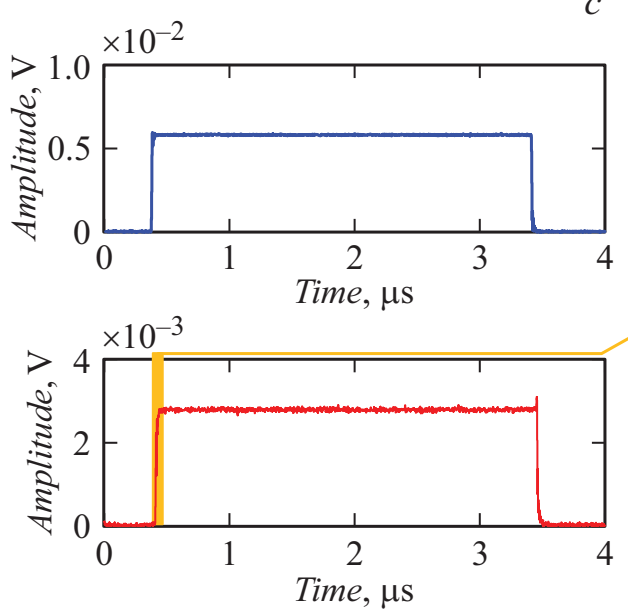
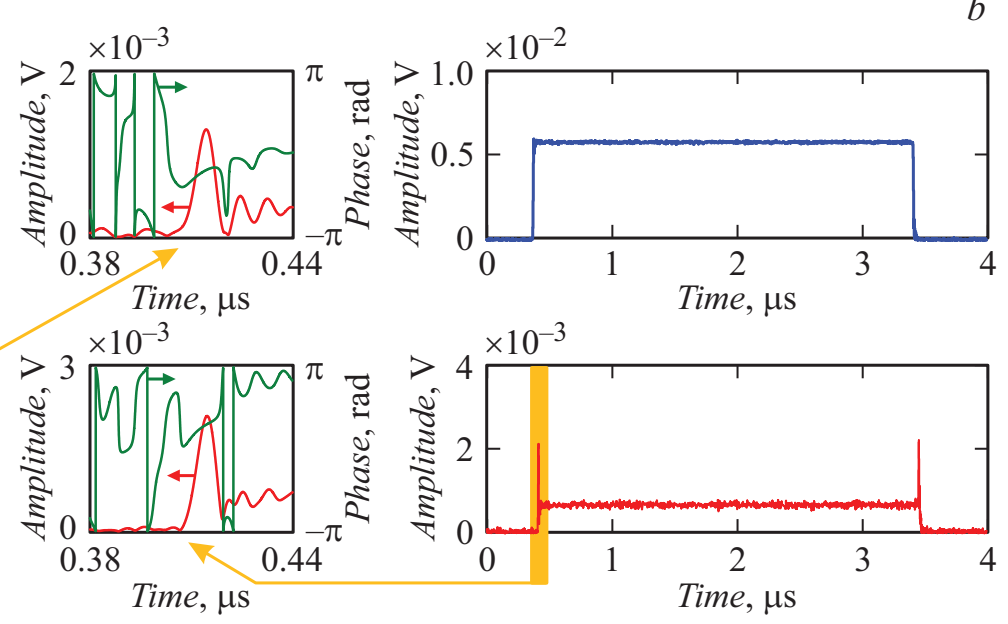

$d$
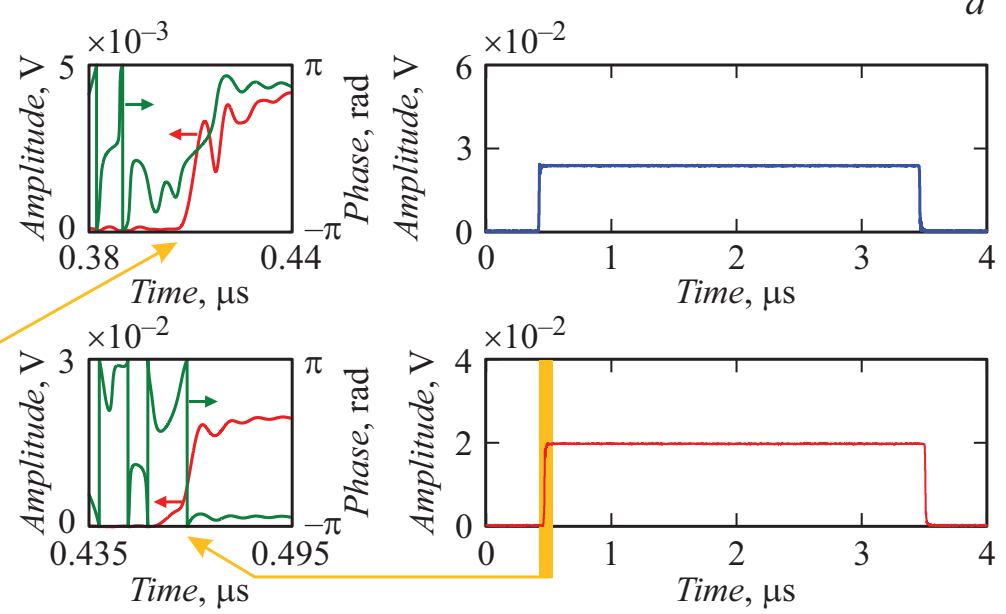

Рис. 2. Огибающие радиоимпульса на входе (верхние фрагменты рисунков $(a)-(c)$ ) и выходе (нижние фрагменты рисунков $(a)-(c))$ ЛБВ подавителя, работающего как насыщающийся поглотитель при различных значениях пиковой мощности $P_{p}$ : $-20(a),-15(b),-9(c)$ и $-3 \mathrm{dBm}(d)$. На вставках показаны увеличенные фрагменты амплитудных и фазовых профилей коротких импульсов огибающей. Измерения проведены при $U_{0}=108 \mathrm{~V}$ и $I_{0}=3 \mu \mathrm{A}$.

в тринадцать раз (рис. 2,c) обусловливает слияние коротких „выплесков“ с основной частью выходного радиоимпульса. При уровнях пиковой мощности, соответствующих максимальному коэффициенту передачи ЛБВ подавителя (рис. 2, $d$ и 1,c), короткие импульсы огибающей полностью сливаются с основной частью выходного радиоимпульса и становятся неразличимыми на его фоне. В то же самое время, как следует из результатов, представленных на рис. $3, c, d$, в режиме нелинейного срыва Компфнера увеличение пиковой мощности входного радиоимпульса не приводит к слиянию „выплесков“ с основной частью выходного радиоимпульса. Напротив, с увеличением пиковой мощности длительность коротких импульсов огибающей уменьшается, т.е. короткие импульсы огибающей испытывают своего рода компрессию при $P_{p}>P_{t h 2}$. Так, при превышении пиковой мощности входного радиоимпульса над порогом $P_{t h 2}$ в 1.4 раза (рис. 3,c) длительность короткого импульса огибающей, находя- щегося на фронте выходного радиоимпульса, уменьшается до $6.2 \mathrm{~ns}$, а при превышении пиковой мощности входного радиоимпульса над порогом $P_{t h 2}$ в 4 раза (рис. $3, d$ ) длительность короткого импульса огибающей уменьшается до $2.7 \mathrm{~ns}$. Отсюда следует, что длительность коротких импульсов огибающей практически линейно зависит от уровня пиковой мощности входного радиоимпульса. При этом, как следует из результатов, представленных на рис. 3, , фаза внутри короткого импульса огибающей имеет практически линейное нарастание во времени, что характерно для импульсных сигналов с линейно-частотной модуляцией [15]. Отметим, что длительность коротких импульсов огибающей определяется временем переключения ЛБВ из линейного режима работы в нелинейный режим. Как показывает эксперимент, это время уменьшается с увеличением мощности входного сигнала, так как уменьшается длительность коротких импульсов огибающей. 
$a$
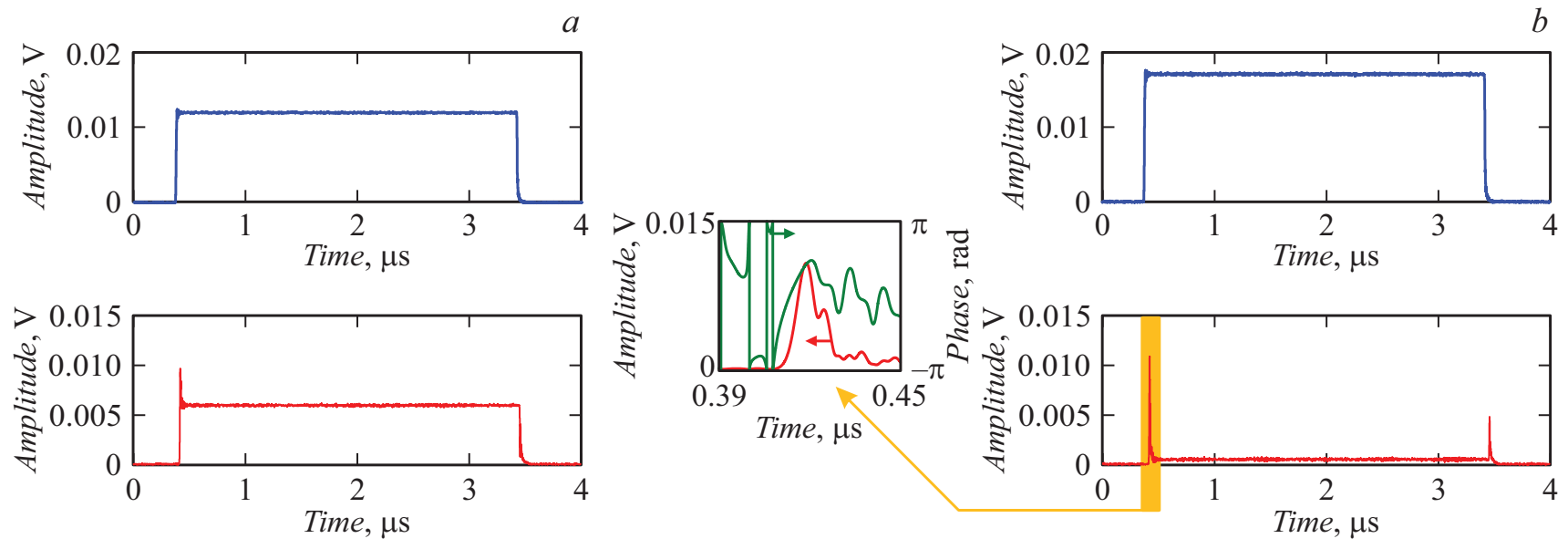

$c$
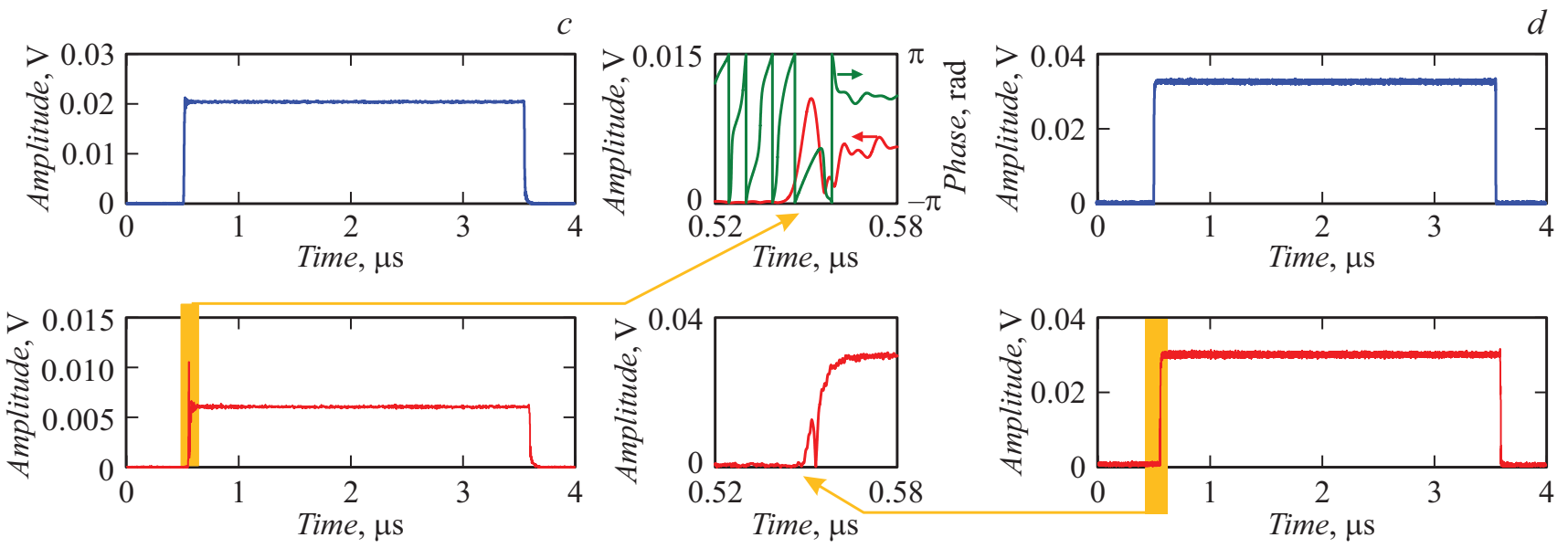

Рис. 3. Огибающие радиоимпульса на входе (верхние фрагменты рисунков $(a)-(c)$ ) и выходе (нижние фрагменты рисунков $(a)-(c))$ ЛБВ подавителя, работающего как ограничитель мощности при различных значениях пиковой мощности $P_{p}:-9.4(a)$, $-6.4(b),-4.9(c)$ и $-0.4 \mathrm{dBm}(d)$. На вставках к рисункам $(b-d)$ показаны увеличенные фрагменты амплитудных и фазовых профилей коротких импульсов огибающей. Измерения проведены при значениях $U_{0}=107 \mathrm{~V}$ и $I_{0}=10 \mu \mathrm{A}$.

Для сравнения были проведены экспериментальные исследования прохождения радиоимпульсов через данную ЛБВ, которая работала в режиме нелинейного усиления ИМ СВЧ сигнала. Исследования были выполнены при схожих значениях ускоряющего напряжения лампы, а также пиковой мощности и длительности входных радиоимпульсов. Установлено, что в режиме нелинейного усиления основная часть радиоимпульса также начинает подавляться, а на его фронте и срезе также образуются короткие „выплески“. Однако в данном случае это происходит из-за ограничения коэффициента усиления лампы, что не приводит к практически полному подавлению основной части выходного радиоимпульса и к отделению от нее „выплесков“ в виде коротких импульсов огибающей. С физической точки зрения это обусловлено тем, что в режиме нелинейного усиления трудно добиться практически полного подавления СВЧ сигнала за счет изменения амплитуды бегущей волны, так как электроны даже при больших уровнях мощности входного СВЧ сигнала по-прежнему находятся в тормозящей фазе поля. Наибольшее ускорение электронного пучка с помощью варьирования амплитуды бегущей волны достигается только в режиме нелинейного срыва Компфнера, когда электроны долгое время находятся в ускоряющей фазе поля при любых уровнях мощности входного сигнала. Именно в этом случае и происходит формирование коротких импульсов огибающей на фронте и срезе выходного радиоимпульса.

\section{2. Нестационарная нелинейная модель ЛБВ подавителя}

\section{1. Основные уравнения}

Для численного моделирования нестационарных процессов воспользуемся традиционной численной моделью, построенной на основе нестационарной нелинейной теории [16,17]. Ранее подобный подход применялся, в частности, для исследования сложной динамики ЛБВ генератора миллиметрового диапазона с запаздывающей обратной связью и замедляющей системой типа „Петляющий волновод“ [18]. Уравнения, описывающие 
взаимодействие электронного пучка с электромагнитной волной, имеют следующий вид:

$$
\begin{aligned}
& \frac{\partial^{2} \theta}{\partial \xi^{2}}=-L^{2}\left(1+\frac{1}{2 \pi N} \frac{\partial \theta}{\partial \xi}\right)^{3} \\
& \times \operatorname{Re}\left[F \exp (i \theta)+i q \sum_{m} \frac{R_{m}^{2} I_{m} \exp (i m \theta)}{m}\right], \\
& \frac{\partial F}{\partial \tau}+\frac{\partial F}{\partial \xi}+d F=-L I_{1},
\end{aligned}
$$

где $\theta=\left(\omega_{0} t-k_{0} x\right)$ - фаза электрона в поле волны, $\omega_{0}$ - частота, на которой скорость электронного пучка $v_{0}$ равна фазовой скорости электромагнитной волны $v_{p h}, k_{0}=\omega_{0} / v_{0}, \xi=x / l$ - безразмерная координата, $l$ - длина пространства взаимодействия, $L=2 \pi C N-$ безразмерный параметр, имеющий смысл нормированной длины пространства взаимодействия, $C=\left[I_{0} R_{c} /\left(4 U_{0}\right)\right]^{1 / 3}, I_{0}$ и $U_{0}-$ ток и напряжение пучка, $N=k_{0} l /(2 \pi)$ - электрическая длина пространства взаимодействия, $q \equiv 4 Q C=\left(\omega_{p} / \omega_{0} C\right)^{2}-$ параметр пространственного заряда, $\omega_{p}-$ плазменная частота, $R_{m}$ - коэффициент редукции плазменной частоты, учитывающий поперечные размеры пролетного канала, $F$ - безразмерная нормированная амплитуда сигнала, $\tau=\left(t-x / v_{0}\right) /\left(l / v_{g}-l / v_{0}\right)-$ безразмерное время, $v_{g}=|\partial \omega / \partial k|-$ групповая скорость волны на частоте $\omega_{0}, d-$ параметр затухания, $I_{1}-$ комплексная амплитуда первой гармоники сгруппированного тока, нормированная на постоянный ток пучка $I_{0}, I_{m}-m$ я гармоника сгруппированного тока, нормированная на постоянный ток пучка $I_{0}$ :

$$
I_{m}=\frac{1}{\pi} \int_{0}^{2 \pi} \exp (-i m \theta) d \theta_{0},
$$

где $\theta_{0}=\theta(x=0)$ - начальная фаза $\left(\theta_{0} \in[0 ; 2 \pi]\right)$. Сформулированные выше уравнения (1)-(3) справедливы только в случае, когда групповая скорость волны меньше ее фазовой скорости. ${ }^{2}$

Уравнения (1)-(3), описывающие динамику системы „электронный поток-электромагнитная волна“, следует дополнить граничными и начальными условиями в виде

$$
\begin{gathered}
\left.\theta\right|_{\xi=0}=\theta_{0},\left.\quad \frac{\partial \theta}{\partial \xi}\right|_{\xi=0}=0, \\
\left.F\right|_{\xi=0}=F_{i n},\left.\quad F\right|_{\tau=0}=F^{0}(\xi) .
\end{gathered}
$$

Условия (4) означают, что на вход волноведущей структуры поступает невозмущенный электронный поток, не модулированный ни по скорости, ни по плотности, а амплитуда поля электромагнитной волны на левой границе системы задана усиливаемым сигналом.

\footnotetext{
2 Это условие выполняется в рабочем режиме исследуемой ЛБВ.
}

Для численного решения нелинейной нестационарной модели в виде уравнений (1)-(4) использовалась модификация хорошо зарекомендовавшего себя конечноразностного метода второго порядка точности $[19,20]$. Выбор данного подхода обусловлен тем, что он широко применяется для теоретического анализа нестационарных процессов в приборах О-типа и имеет аналогичные управляющие параметры.

\section{2. Результаты численного моделирования}

\subsection{1. Нелинейное подавление монохроматического сигнала}

Нелинейное подавление монохроматического сигнала в ЛБВ исследовалось с использованием одномерной стационарной многочастотной нелинейной теории ЛБВ [21]. Электродинамические параметры спиральной 3С рассчитывались по методике, описанной в [22]. Моделирование нелинейного подавления сигнала в стационарном режиме проводилось с использованием модуля TWT1D из программы TWT3D [23]. При этом величины, соответствующие экспериментальным параметрам, а также рассчитанные выходные характеристики лампы представлялись непосредственно в размерном виде. Кроме того, были определены значения безразмерных параметров лампы, которые использовались в дальнейшем в нелинейной нестационарной модели.

На рис. 4 приведены результаты численного моделирования зависимостей коэффициента передачи ЛБВ подавителя как от частоты, так и от уровня мощности входного сигнала. Моделирование проводилось для двух значений тока пучка и ускоряющего напряжения, соответствующих режиму срыва Компфнера (рис. 4, a, c) и режиму нелинейного срыва Компфнера (рис. 4, $b, c$ ). Из представленных на рис. 4, $a$ результатов расчета следует, что при малых уровнях входной мощности сигнала (линейный режим) на зависимости $K(f)$ наблюдается глубокий провал, центральная частота которого $f_{01} \cong 792 \mathrm{MHz}$ близка к частоте $f_{0}$. Увеличение мощности приводит к уменьшению глубины провала, к сдвигу его центральной частоты вниз по частоте и к его полному исчезновению при больших значениях входной мощности. В эксперименте аналогичный эффект наблюдался в режиме срыва Компфнера (рис. 1,a). На рис. 4, $a$ штриховой линией показана АЧХ ЛБВ подавителя, рассчитанная в линейном режиме, но при значении тока пучка, превышающем предыдущее значение на величину $\sim 0.4 \mu \mathrm{A}$. Видно, что плавная настройка величины тока пучка в численном моделировании позволяет добиться практически бесконечного уровня подавления сигнала, что не удавалось достичь в эксперименте из-за грубой регулировки величины тока. Необходимо отметить, что расхождение в $5.3 \mathrm{~dB}$ между уровнями входной мощности, при которых в эксперименте и численном моделировании наблюдается максимальный уровень подавления сигнала, связано, по всей видимости, с неидеальностью 

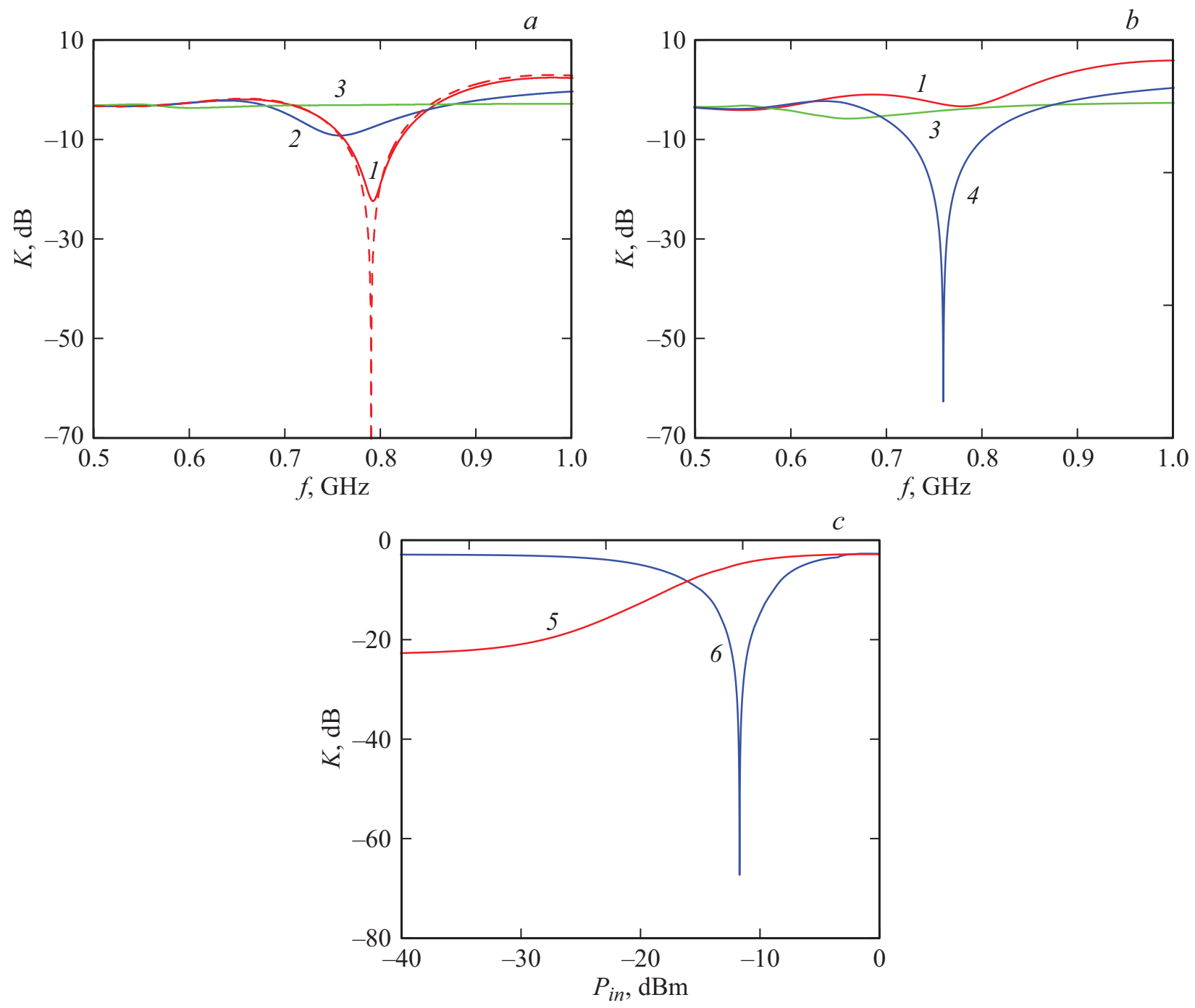

Рис. 4. $a, b-\mathrm{AЧX} \mathrm{ЛБВ,} \mathrm{рассчитанные} \mathrm{в} \mathrm{режиме} \mathrm{срыва} \mathrm{Компфнера}\left(I_{0}=3 \mu \mathrm{A}, U_{0}=108 \mathrm{~V}\right)(a)$ и в режиме нелинейного срыва Компфнера $\left(I_{0}=10 \mu \mathrm{A}, U_{0}=107 \mathrm{~V}\right)(b)$, при разных значениях входной мощности $P_{i n}:-35.3$ (кривая 1$),-15.3$ (кривая 2), -5.3 (кривая 3) и $-11.7 \mathrm{dBm}$ (кривая 4); $c$ - зависимости модуля коэффициента передачи $K$ от мощности сигнала на выходе ЛБВ подавителя $P_{i n}$, рассчитанные на частоте $f_{01}$ в режиме срыва Компфнера (кривая 5 ), и на частоте $f_{02}$ в режиме нелинейного срыва Компфнера (кривая 6). На $a$ штриховой линией показана АЧХ, рассчитанная для $I_{0}=3.4259807 \mu \mathrm{A}$ и $U_{0}=108 \mathrm{~V}$.

моделирования системы ввода/вывода энергии лампы из-за отсутствия о ней точной технической информации.

На рис. 4,c (кривая 5) представлена зависимость $K\left(P_{i n}\right)$, рассчитанная на частоте $f_{01}$ в режиме срыва Компфнера, которая демонстрирует изменение коэффициента передачи ЛБВ-подавителя с ростом входной мощности. Видно, что, как и в эксперименте (рис. 1,c), при больших уровнях входной мощности коэффициент передачи является максимальным. С уменьшением уровня входной мощности он уменьшается и в линейном режиме становится минимальным (насыщается). Таким образом, результаты численного моделирования подтверждают тот факт, что в режиме срыва Компфнера при изменении амплитуды электромагнитной волны ЛБВ подавитель работает как насыщающийся поглотитель.
На рис. 4, b, c приведены результаты численного моделирования частотных и амплитудных зависимостей модуля коэффициента передачи ЛБВ подавителя, рассчитанные для режима нелинейного срыва Компфнера. Из результатов расчета следует, что при выбранных значениях тока и напряжения пучка режим практически полного подавления сигнала наблюдается уже не в линейном, а в нелинейном режиме, на частоте $f_{02} \cong 760 \mathrm{MHz}$. Здесь, так же как и в эксперименте (рис. $1, b)$, минимальный уровень подавления сигнала наблюдается в линейном режиме, а практически полное подавление сигнала происходит только при определенном уровне мощности. Такое избирательное по мощности подавление сигнала приводит к тому, что ЛБВ-подавитель работает как ограничитель мощности (ср. рис. 4, с и 1, с- кривые 6). 

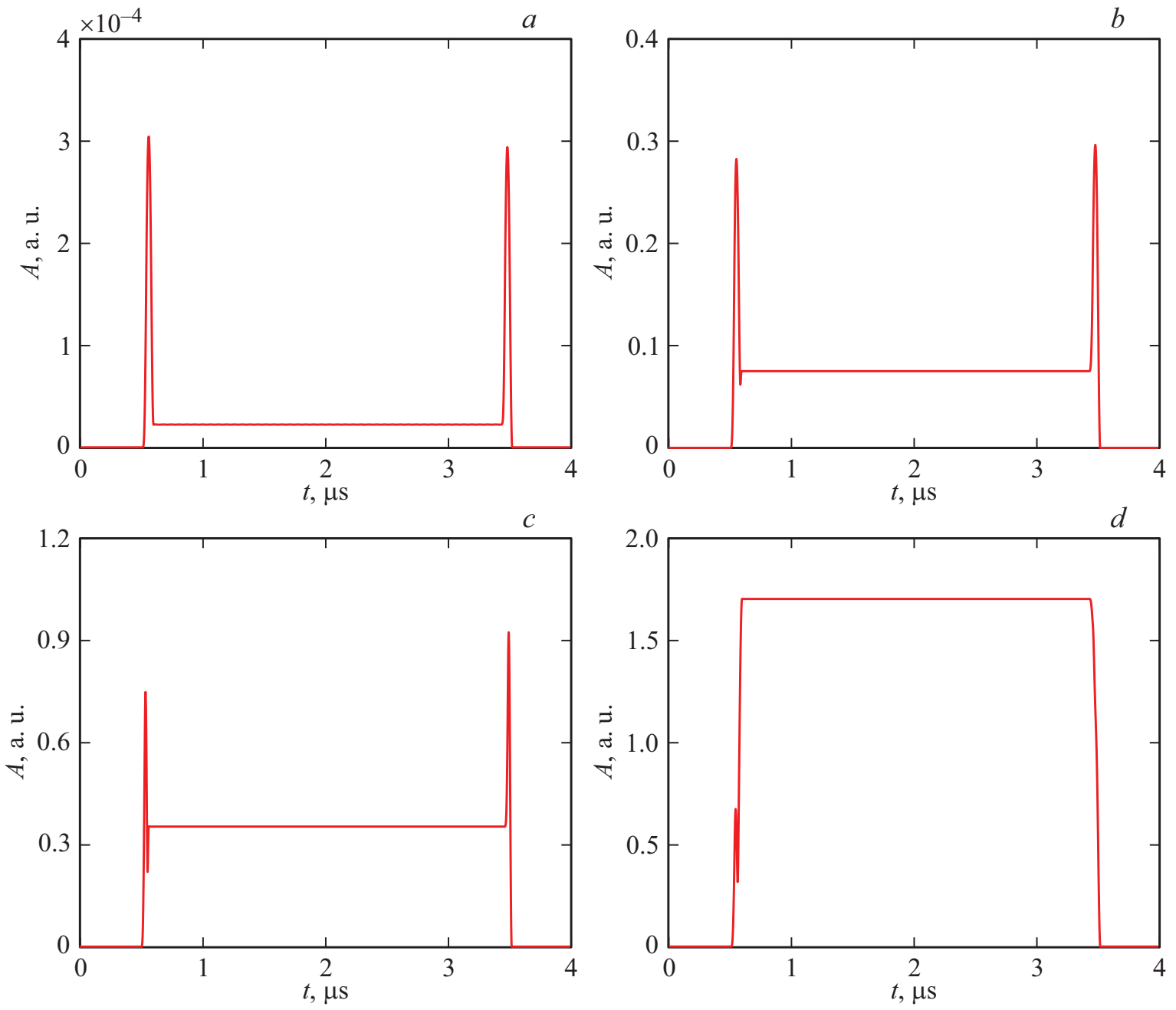

Рис. 5. Рассчитанные по нестационарной нелинейной теории огибающие радиоимпульса на выходе ЛБВ подавителя, работающего как насыщающийся поглотитель при различных значениях пиковой мощности $P_{p}$ : $-80(a),-20(b),-15(c)$ и $-9 \mathrm{dBm}(d)$.

\subsection{2. Нелинейное подавление импульсного сигнала}

Помимо численного моделирования режимов подавления монохроматического сигнала было проведено численное моделирование прохождения импульсного сигнала разного уровня мощности через ЛБВ подавитель, работающий в режиме срыва Компфнера и в режиме нелинейного срыва Компфнера. Для этих целей использовалась нелинейная нестационарная модель в виде уравнений (1)-(4). При моделировании использовался импульс со сглаженным фронтом, характеризующийся временем нарастания порядка $60 \mathrm{~ns}$ (при пересчете в размерные величины). Безразмерные параметры модели выбирались близкими к экспериментальным данным. Так, электрическая длина пространства взаимодействия бралась равной $N=24.15$, параметр затухания $d=0.345$, а параметр пространственного заряда $Q=3.173$. Моделирование проводилось для двух значений тока пучка и ускоряющего напряжения, которым соответствовали два значения $C N$ : 0.3 - режим срыва Компфнера и 0.45 - режим нелинейного срыва Компфнера. Результаты моделирования нестационарных процессов приведены на рис. 5 и 6.

Из представленных на рис. 5, $a$ результатов следует, что в режиме срыва Компфнера наблюдается практически полное подавление основной части импульса, на фронте и срезе которого остаются „выплески“ малой длительности, что наблюдалось ранее в эксперименте (рис. 2,a). По мере увеличения входной мощности импульса (рис. 5, $b, c$ ) подавление его основной части уменьшается, и она начинает подрастать вместе с амплитудами „выплесков“. При достаточно больших уровнях пиковой мощности входного импульса (рис. 5, $d$ ) срезы „выплесков“, как и в эксперименте, практически полностью сливаются с основной частью выходного импульса.

В режиме нелинейного срыва Компфнера (рис. 6) численное моделирование демонстрирует схожее с экспериментом поведение выходного импульса при изменении его входной мощности (рис. 3). Как следует из результатов, представленных на рис. 6, $a$, подавление 

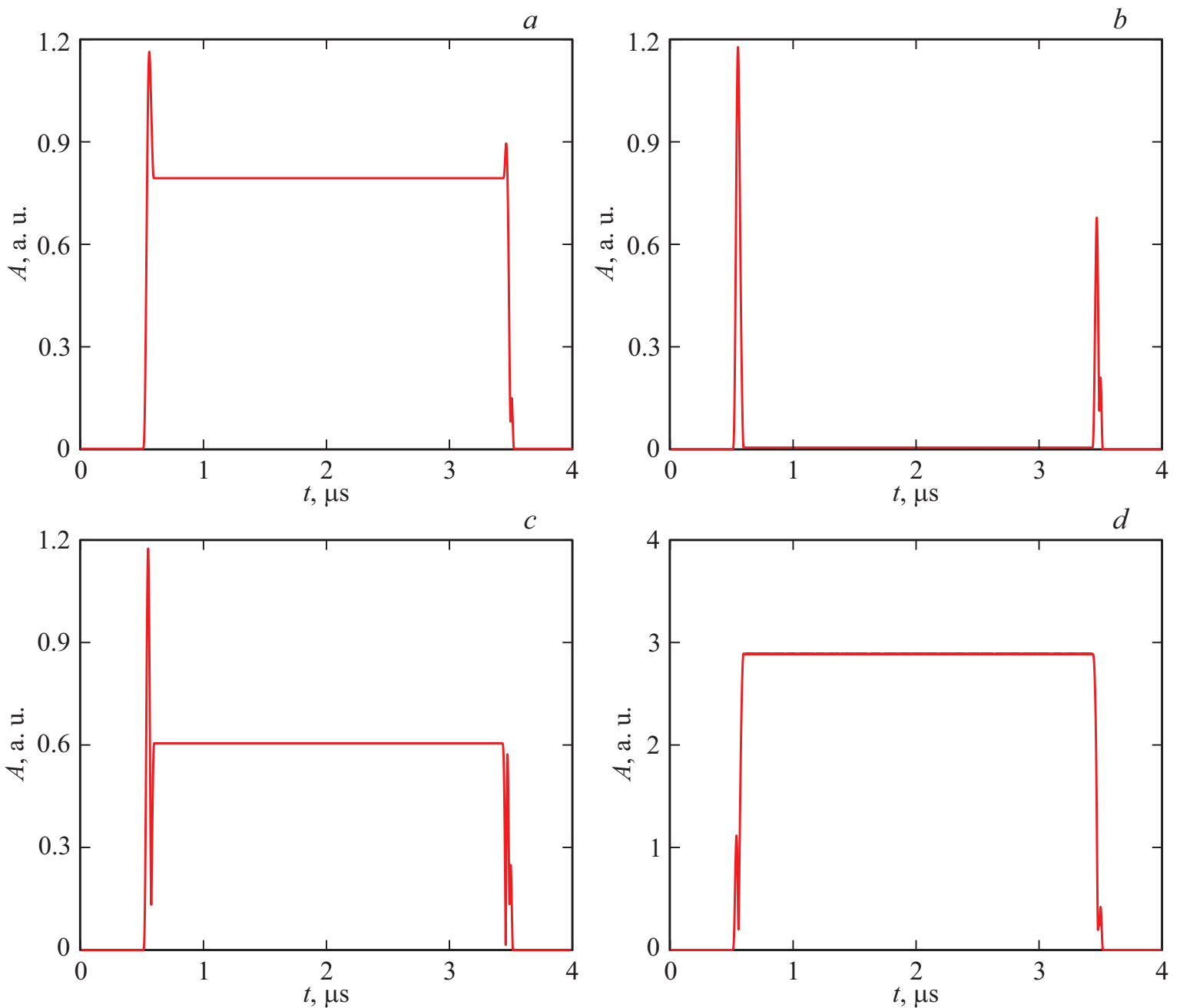

Рис. 6. Рассчитанные по нестационарной нелинейной теории огибающие радиоимпульса на выходе ЛБВ подавителя, работающего как ограничитель мощности при различных значениях пиковой мощности $P_{p}:-9.4(a),-6.4(b),-4.9(c)$ и $-0.4 \mathrm{dBm}(d)$.

основной части импульса и образование на его фронте и срезе „выплесков“ в виде коротких импульсов огибающей происходит только при переходе в нелинейный режим. Практически полное подавление основной части импульса и образование коротких импульсов огибающей на его фронте и срезе, как и в эксперименте, регистрируется при уровне входного сигнала, значительно превышающем аналогичный уровень, соответствующий режиму линейного подавления (рис. $6, b$ ). При дальнейшем увеличении входной мощности импульса (рис. $6, c, d$ ), хотя длительность короткого „выплеска“ на фронте импульса уменьшается практически в 1.5 раза, однако срез короткого „выплеска“ начинает сливаться с основной частью выходного импульса. Данный факт, может быть, обусловлен тем, что используемая нелинейная нестационарная модель пригодна, в основном, для моделирования узкополосных процессов, характерный временной масштаб которых ограничен снизу временем распространения огибающей сигнала по 3С.

\section{Заключение}

В заключение отметим, что в работе продемонстрирована возможность формирования импульсов огибающей наносекундной длительности с помощью ЛБВ подавителя, у которого максимальный уровень затухания СВЧ сигнала наблюдается либо в линейном режиме (классический срыв Компфнера), либо в нелинейном режиме (нелинейный срыв Комфнера). Показано, что наименьшей длительностью обладают импульсы огибающей, полученные в режиме нелинейного срыва Компфнера. В практическом плане полученные результаты могут быть полезными при разработке источников импульсных сигналов для систем связи и локации.

\section{Финансирование работы}

Работа выполнена за счет гранта Российского фонда фундаментальных исследований (проект № 18-0200666). 


\section{Конфликт интересов}

Авторы заявляют, что у них нет конфликта интересов.

\section{Список литературы}

[1] Д.И. Трубецков, Г.М. Вдовина. УФН, 190 (5), 543 (2020).

[2] R. Kompfner. J. British IRE, 10 (8-9), 283 (1950).

[3] H.R. Johnnson. PIRE, 7, 874 (1955).

[4] В.Н. Шевчик, Д.И. Трубецков. Аналитические методы расчета в электронике СВЧ (Сов. радио, М., 1970)

[5] D.A. Watkins, A.E. Siegman. J. Appl. Phys., 7, 917 (1953).

[6] R.W. Gould. IRE Trans., ED-2 (4), 37 (1955).

[7] A. Ashkin, W.H. Louisell, C.F. Quate. J. Electronics and Control. 7, 1 (1959).

[8] Б.С. Дмитриев, Ю.Д. Жарков. В сб.: Сборник докладов секиии электроники XXI Всесоюзной научной сессии НТОРЭ (М., 1965), с. 37.

[9] Б.С. Дмитриев, Ю.Д. Жарков. В сб.: Вопросы электроники сверхвысоких частот (Сарат. ун-т, Саратов, 1966), B. 2 , с. 3 .

[10] Ю.А. Калинин, Ю.А. Григорьев, В.К. Семенов. Обзоры по электронной технике. Серия 1. Электроника СВЧ., 2 (1329), 48 (1988).

[11] Н.С. Гинзбург, Э.Б. Абубакиров, М.Н. Вилков, И.В. Зотова, А.С. Сергеев. Письма в ЖТФ, 43 (18), 47 (2017). DOI: 10.21883/PJTF.2017.18.45033.16444b

[12] N.S. Ginzburg, G.G. Denisov, M.N. Vilkov, A.S. Sergeev, I.V. Zotova, S.V. Samsonov, S.V. Mishakin. Phys. Plasmas, 24 (2), 023103 (2017). DOI: 10.1063/1.4975084

[13] Н.С. Гинзбург, Э.Б. Абубакиров, М.Н. Вилков, И.В. Зотова, А.С. Сергеев. ЖТФ, 88 (8), 1241 (2018). DOI: 10.21883/JTF.2018.08.46316.2581

[14] S.V. Grishin, B.S. Dmitriev, O.I. Moskalenko, V.N. Skorokhodov, Yu.P. Sharaevskii. Phys. Rev. E, $98(2)$, 022209 (2018). DOI: 10.1103/PhysRevE.98.022209

[15] И.С. Гоноровский. Радиотехнические цепи и сигналы (Сов. радио, М., 1977)

[16] Ю.П. Блиох, М.Г. Любарский, В.О. Подобинский, Я.Б. Файнберг. Физика плазмы, 20 (7-8), 718 (1994).

[17] Yu.P. Bliokh, M.G. Liubarskii, V.O. Podobinskii, Ya.B. Fainberg, G.S. Nusinovich, S. Kobayashi, Y. Carmel, V.L. Granatstein. Phys. Plasmas, 5 (11), 4061 (1998). DOI: $10.1063 / 1.873128$

[18] N.M. Ryskin, V.N. Titov, S.T. Han, J.K. So, K.H. Jang, G.S. Park. Phys. Plasmas, 11 (3), 1194 (2004). DOI: $10.1063 / 1.1640622$

[19] Н.С. Гинзбург, С.П. Кузнецов, Т.Н. Федосеева. Изв. вузов. Радиофизика, 21 (7), 1037 (1978).

[20] Б.П. Безручко, Л.В. Булгакова, С.П. Кузнецов, Д.И. Трубецков. В сб. Лекции по электронике СВЧ и радиофизике (5-я зимняя школа-семинар инженеров). Кн. 5. (Сарат. ун-т, Саратов, 1980), с. 25.

[21] А.М. Кац, Е.М. Ильина, И.А. Манькин. Нелинейные явления в СВЧ приборах О-типа с длительным взаимодействием (Сов. радио, М., 1975)

[22] D. Chernin, T.M. Antonsen, B. Levush. IEEE Trans. on ED, 46 (7), 1472 (1999). DOI: 10.1109/16.772493

[23] A.G. Rozhnev, N.M. Ryskin, D.V. Sokolov, D.I. Trubetskov, A.S. Pobedonostsev, S.A. Rumyantsev, V.B. Khomitch. Fifth IEEE International Vacuum Electronics Conference (IVEC) (Monterey, 2004), p. 144.

DOI: 10.1109/IVELEC.2004.1316240 